\title{
Anakinra and related drugs targeting interleukin-I in the treatment of cryopyrin- associated periodic syndromes
}

This article was published in the following Dove Press journal:

Open Access Rheumatology: Research and Reviews

3 March 2014

Number of times this article has been viewed

\author{
Inessa Bachove' \\ Christopher Chang ${ }^{2}$ \\ 'Cooper University Hospital, \\ Children's Regional Hospital, Camden, \\ NJ, USA; ${ }^{2}$ Division of Allergy and \\ Immunology, Thomas Jefferson \\ University, Wilmington, DE, USA
}

\begin{abstract}
Anakinra is an interleukin (IL) receptor antagonist that works by blocking the biological activity of IL-1 by competitively inhibiting binding of IL-1 to the type 1 interleukin receptor. IL-1 production is induced in response to inflammatory stimuli and mediates various physiological mechanisms, including inflammation and immunological reactions. Patients with neonatal onset multisystem inflammatory disease (NOMID) produce excess IL-1 $\beta$, a major proinflammatory cytokine that regulates innate immune responses. Anakinra binds competitively and this results in a rapid reduction in disease severity. NOMID, also known as chronic infantile neurologic, cutaneous, articular syndrome, is the most severe clinical phenotype in the spectrum of cryopyrin-associated periodic syndromes. It is characterized by cutaneous symptoms, arthropathy, and central nervous system involvement. Extensive studies in patients with NOMID have led to advances in characterizing the extent of organ-specific involvement and damage that occurs with chronic overproduction of IL-1 $\beta$. NOMID is caused predominantly by mutations in the NLRP3/CIAS1 gene that encodes for the protein cryopyrin, leading to activation of the "NLRP3 inflammasome complex". This in turn regulates the maturation and secretion of the inflammatory cytokine, IL-1 $1 \beta$. The clinical value of IL-1 $\beta$ has been demonstrated by the positive response of patients after treatment with anakinra, with rapid improvement in clinical symptoms, markers of inflammation, and a significant decrease in major organ manifestations.
\end{abstract}

Keywords: neonatal onset multisystem inflammatory disease, chronic infantile neurologic, cutaneous, arthritis syndrome, NLRP3, CIAS1, interleukin-1 $\beta$

\section{Introduction}

Neonatal onset multisystem inflammatory disease (NOMID) is a monogenic autoinflammatory syndrome and constitutes one of the three known cryopyrin-associated periodic syndromes (CAPS). These syndromes occur as a result of an autosomal dominant or de novo mutation of the cold-induced autoinflammatory syndrome 1 (CIAS1) gene on chromosome 1q44. ${ }^{1}$ The three clinical entities that belong to the group of diseases known as CAPS were discovered at different times, and initially described separately as distinct diseases. It was not until a common genetic defect was identified that these three diseases were recognized as separate clinical phenotypes of the same disease mechanism. The three forms of CAPS are familial cold autoinflammatory syndrome (FCAS), Muckle-Wells syndrome (MWS), and NOMID, or as it is known in Europe, chronic infantile neurological, cutaneous, articular (CINCA) syndrome. The discovery of a common etiology for these diseases is an important step in the understanding of autoinflammatory disease. The clinical spectrum, including both similarities and differences in presentation of the three diseases, adds to the intrigue surrounding the
Correspondence: Christopher Chang Division of Allergy and Immunology, Thomas Jefferson University, 1600 Rockland Road, Wilmington, DE 19803, USA

$\mathrm{Tel}+|30265| 432$ I

Fax +| 30265 | 6558

Email cchang@nemours.org
Open Access Rheumatology: Research and Reviews 20 I4:6 I5-25 BY LC License. The full terms of the License are available at http://creativecommons.org/licenses//by-n/3.0/. Non-commercial uses of the work are permitted without any further (c) (i) () 2014 Bachove and Chang. This work is published by Dove Medical Press Limited, and licensed under Creative Commons Attribution - Non Commercial (unported, v3.0)

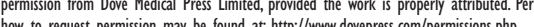
how to request permission may be found at: http://www.dovepress.com/permissions.php 
common genetic defect. Why does a single common defect lead to such diverse clinical symptoms and signs?

Along the clinical spectrum of CAPS, all three diseases present in the neonatal period, although there have been reports of presentation after infancy and even into adulthood. Common features of these diseases include inflammation of the skin, eyes, bones, joints, and meninges. In addition, severe fatigue, fever, myalgia, chronic anemia, and learning disabilities are some of the other symptoms which are commonly seen. ${ }^{2}$ The similarities and differences of these diseases are shown in Table 1. These diseases make up a group of rare hereditary autoinflammatory diseases, with an estimated population frequency in the range of 1-3 per million. $^{3}$

The discovery of a genetic basis for these disorders has significantly impacted new approaches to treatment. The CIAS1 gene, which is mutated in CAPS patients, has been discovered to be involved in inflammation and apoptosis. ${ }^{4}$ In response to inflammation, interleukin (IL)- $1 \beta$ is secreted and is known to mediate the peripheral immune response during infection and inflammation. It has been reported that patients with NOMID and other CAPS disorders have higher IL-1 $\beta$ levels compared with their healthy counterparts. Thus, the mutation in CAPS is a gain-of-function mutation.

As a result of the pathogenesis, targeting the IL-1 $\beta$ pathway has proven to be a successful approach in the treatment of patients with CAPS, specifically NOMID. Recent advances in the development of drugs targeting IL-1 $\beta$ have led to the introduction of several anti-IL-1 $\beta$ drugs, including anakinra, rilonacept, and canakinumab.

\section{Cryopyrin-associated periodic syndromes}

The three cryopyrin-associated periodic syndromes are FCAS, MWS, and NOMID. Chronic inflammation, with recurrent fever, cutaneous rash, and joint symptoms, are common characteristics of these diseases. As they progress from the mildest form to the most severe form, the rash becomes less prominent, and other more severe features dominate the clinical presentation. ${ }^{5}$ This spectrum of disease severity is shown in Table 1.

\section{Familial cold autoinflammatory syndrome}

FCAS is the mildest phenotype of the autoinflammatory diseases and is characterized by urticarial rash, low grade fever, polyarthralgias, and conjunctivitis induced by exposure to cold, occurring primarily in infancy. ${ }^{6}$ These episodes are generally self-limiting and resolve within 24 hours. Other symptoms induced by cold exposure include profuse sweating, drowsiness, headache, extreme thirst, and nausea. ${ }^{7}$ Although these symptoms have a significant impact on quality of life, they are not associated with organ damage or disability. Laboratory studies show elevated neutrophil counts, erythrocyte sedimentation rate, and $\mathrm{C}$-reactive protein.

\section{Muckle-Wells syndrome}

MWS is the intermediate phenotype and is characterized by recurrent episodes of rash and fever that may develop in the first few years of life. The rash can be cold-induced, urticarial, or erythematous. Musculoskeletal symptoms include arthralgia, arthritis, and myalgias. These features occur episodically and can last anywhere from one day to 2 weeks.

Table I Similarities and differences between CAPS with symptom progression from the mildest form to the most severe form of the disease

\begin{tabular}{|c|c|c|c|}
\hline & FCAS & MWS & NOMID/CINCA \\
\hline Severity & Low & Medium & High \\
\hline Triggers & Cold-induced & None & None \\
\hline Fever/rash & Usually daily symptoms & Variable symptoms (rare to daily) & Variable symptoms (rare fever, daily rash) \\
\hline Joint involvement & Arthralgia & Arthralgia/arthritis & Arthralgia, arthritis, overgrowth arthropathy \\
\hline Neurological involvement & None & None & $\begin{array}{l}\text { Chronic aseptic meningitis (headache, } \\
\text { mental retardation) }\end{array}$ \\
\hline Eye involvement & Conjunctivitis & Conjunctivitis, uveitis & Uveitis, papillary edema, optic neuritis \\
\hline Hearing loss & None & Frequent $(60 \%)$ & Frequent $(>60 \%)$ \\
\hline Amyloidosis & No & Frequent $(25 \%)$ & Frequent (25\%) \\
\hline Inheritance & Autosomal dominant & $\begin{array}{l}\text { Autosomal dominant or } \\
\text { de novo (rare) }\end{array}$ & $\begin{array}{l}\text { De novo (typical) or autosomal dominant } \\
\text { (rare) }\end{array}$ \\
\hline Pathophysiology & $\begin{array}{l}\text { Mutation in CIASI gene } \\
\text { encoding for cryopyrin }\end{array}$ & $\begin{array}{l}\text { Mutation in CIASI gene encoding } \\
\text { for cryopyrin }\end{array}$ & $\begin{array}{l}\text { Mutation in CIASI gene encoding for } \\
\text { cryopyrin }\end{array}$ \\
\hline Treatment & Anti-IL-I therapy & Anti-IL-I therapy & Anti-IL-I therapy \\
\hline
\end{tabular}

Abbreviations: IL, interleukin; FCAS, familial cold autoinflammatory syndrome; MWS, Muckle-Wells syndrome; NOMID, neonatal onset multisystem inflammatory disease; CINCA, chronic infantile, neurological, cutaneous, articular syndrome; CAPS, cryopyrin-associated periodic syndromes. 
Sequelae of MWS may include sensorineural hearing loss and secondary renal amyloidosis with nephropathy (a complication of late stage disease).$^{8}$ The hearing loss is progressive, likely leading to deafness by adolescence, and the amyloidosis may progress to impaired renal function. Laboratory findings include generalized leukocytosis and elevations in acute-phase reactants.

\section{Neonatal onset multisystem inflammatory disease}

NOMID is the most severe phenotype, with the course of disease being more chronic than recurrent. It manifests in the first few weeks of life with an urticarial-like rash, arthropathy, and central nervous system involvement. ${ }^{9}$ The rash is typically nonpruritic and can vary with disease activity. Fever is usually mild in this disease. Individuals have atypical "facies", including frontal bossing, a saddle-back nose, and mid-face hypoplasia. The disease also causes bony overgrowths predominantly involving the knees and the distal extremities of the hands and feet. Chronic inflammatory polyarthritis may also be present, resulting in bone erosions. The severity of bone and joint involvement is variable, with approximately two thirds of patients developing arthralgia and transient swelling during flares, and the remaining one third developing severe and disabling arthropathy, resulting in gross deformity of the patella and epiphyses of the long bones. ${ }^{10}$

The radiographic findings in severe cases are characterized by a "bread crumb" appearance of the patella and epiphyses. Central nervous system manifestations can be the most devastating, and include chronic septic meningitis, increased intracranial pressure, cerebral atrophy, ventriculomegaly, sensorineural hearing loss, and chronic papilledema with associated optic nerve atrophy and loss of vision. Mental retardation and seizures are also present. ${ }^{9}$

Findings suggestive of an ongoing inflammatory process include lymphadenopathy, splenomegaly, elevations in acute-phase reactants (erythrocyte sedimentation rate and C-reactive protein), leukocytosis, cerebrospinal fluid neutrophilia, hyperglobulinemia, and chronic anemia. Failure to control the inflammation can lead to irreversible organ damage and disability. Therefore, it is imperative to start treatment as soon as the disease is suspected.

\section{Pathophysiology of autoinflammatory syndromes Genetics of cryopyrin}

The discovery of a genetic basis for NOMID has led to its placement among the other cryopyrinopathies, all of which are caused by mutations in the CIAS1 gene. The genetic defect was first defined in FCAS in 2000, and subsequently the same gene was found to be responsible for MWS. ${ }^{7,11}$ The genetic defect was identified in NOMID in 2002. There are many mutations that lead to CAPS, and are located in exon 3 of the CIAS1 gene. All are gain-of-function mutations and are essential to the activation of intracellular caspase- 1 and processing of IL-1 $1 \beta .^{12}$

\section{Mechanism of action of cryopyrin}

CIAS1 encodes the cryopyrin molecule, which combines with a group of interacting proteins to form a macromolecular complex called the "inflammasome," that is important in the innate immune response due to its role as a molecular platform. ${ }^{13}$ There are at least four inflammasomes, designated NLRP1, NLRP3, NLRC4, and AIM2. ${ }^{14}$ The NLRP3 inflammasome, which is directly responsible for CAPS, contains three separate components that are all involved in the activation of proinflammatory cytokines, such as IL-1 $\beta$ and IL-18. These components include NLRP3/cryopyrin, an apoptosis-associated speck-like protein (ASC) containing a CARD (caspase activation and recruitment domain) and a leucine-rich repeat domain.

The signals leading to inflammasome activation and generation of proinflammatory cytokines such as IL-1 $\beta$ include danger-associated molecular patterns and pathogen-associated molecular patterns. There have been many danger-associated molecular patterns described, including cholesterol or uric acid, toxins, adenosine triphosphate (ATP), and ultraviolet B radiation. ${ }^{15-17}$ Pathogen-associated molecular patterns that have been shown to activate NALP3 include bacteria such as Neisseria gonorrhea and viruses such as Influenza A. ${ }^{18,19}$ Once NLRP3 is stimulated, it oligomerizes and binds the adaptor protein ASC. ASC interacts with cryopyrin, leading to activation of nuclear factor kappa B and caspase-1, and apoptosis. ${ }^{20}$

Activation of caspase- 1 results in cleavage of pro-IL-1 $\beta$ and pro-IL-18 into their mature forms (IL-1 $\beta$ and IL-18, respectively) which are secreted by the cell. ${ }^{21}$ Thus, activated cryopyrin induces release of the active form of IL-1 $1 \beta$. This process is illustrated in Figure 1. In all three phenotypes of CAPS, the gain-of-function mutation of the NLRP3 gene forms a defect in this pathway, resulting in increased inflammasome activation, which enhances and accelerates production of IL-1 $\beta$, leading to the inflammatory symptoms seen in NOMID. ${ }^{22}$

\section{Therapeutic targets and strategies}

The disorders caused by CIAS1 mutations are not distinct diseases, but represent a clinical continuum of phenotypes. 


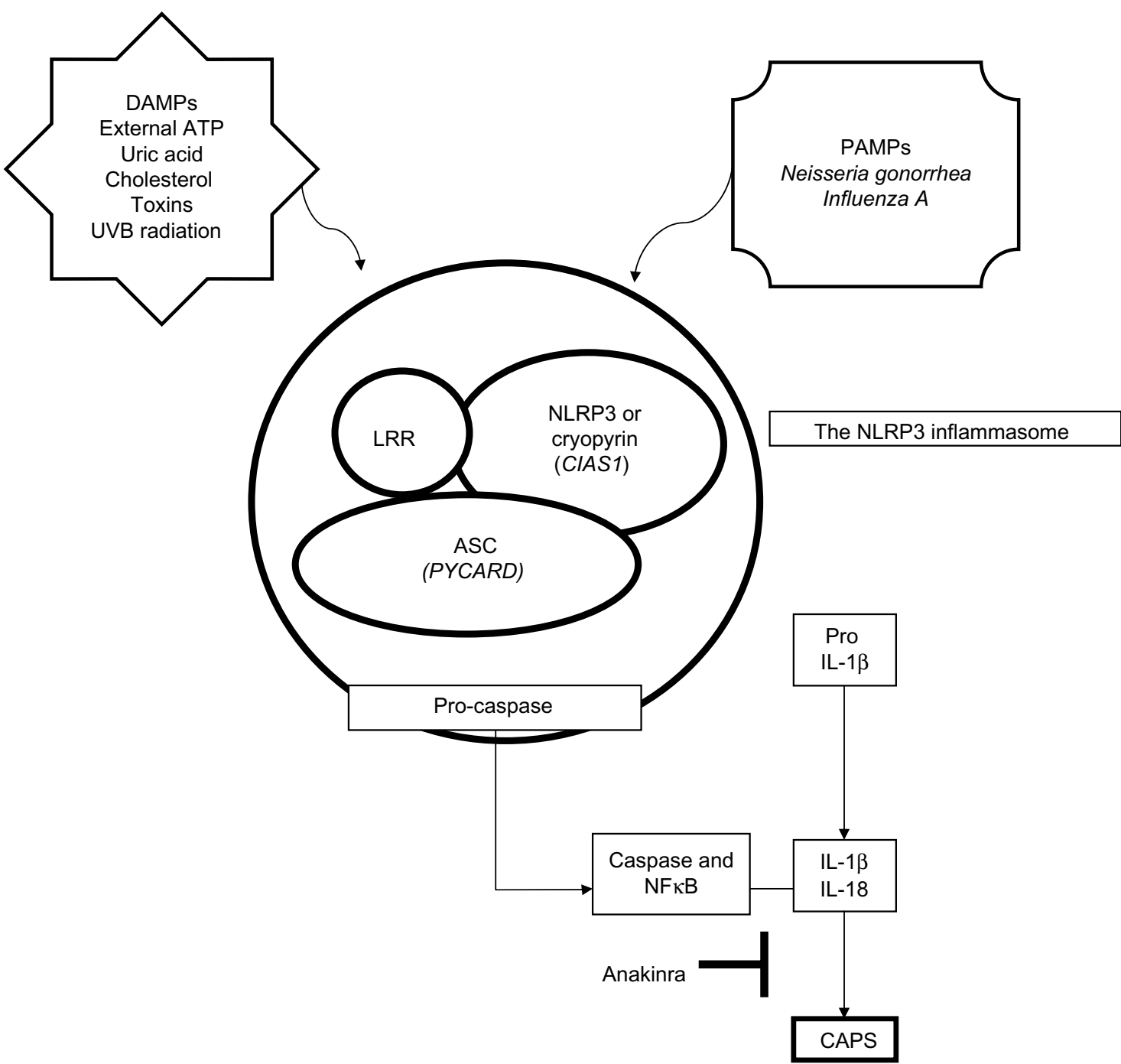

Figure I Pathophysiology of CAPS. Activation of the NLRP3 inflammasome is triggered by exposure of immune cells to a variety of danger-associated molecular patterns and pathogen-associated molecular patterns. The leucine-rich repeat domain of the NLRP3 is thought to serve as an autoinhibitor by self-folding. The molecule spreads out, dimerizes, and associates through homotypic interaction with the ASC adaptor protein to mediate the proteolytic processing of pro-caspase I to caspase-I. The ASC protein also interacts with cryopyrin. When cryopyrin binds to ASC it can result in NFKB and caspase-I activation. Once caspase-I is activated, it results in cleavage of pro-IL-I $\beta$ and pro-IL- 18 into their mature forms IL-I $\beta$ and IL- I8, respectively, which is secreted by the immune cell. Thus, activated cryopyrin induces release of the active form, IL-I $\beta$. Abbreviations: DAMPs, danger-associated molecular patterns; PAMPs, pathogen-associated molecular patterns; IL, interleukin; ASC, apoptosis-associated speck-like protein; CAPS, cryopyrin-associated periodic syndromes; UVB, ultraviolet B; LRR, leucine-rich repeat; ATP, adenosine triphosphate.

It was discovered that the same nucleotide substitution in CIAS1 may be associated with a different clinical subtype in different patients. ${ }^{23}$ It is also interesting that various mutations in CIAS1 result in different levels of caspase-1 activation, thereby leading to such extreme clinical spectrum ranges as seen in the different phenotypes of CAPS. ${ }^{24}$ In addition, CIAS1 mutations may not always be the culprit in diseases involving cryopyrin. This has been evident in several patients with FCAS, MWS, and NOMID who do not have mutations in CIAS1, but have the classic disease phenotype.

Most CIAS1 mutations are inherited in an autosomal dominant pattern, although de novo cases have been reported. Mutations in CIAS1 were found in 50\% of NOMID patients and mutations occurred de novo in five of seven patients with NOMID syndrome. ${ }^{25}$ This suggests that the severity and presence of the disease is likely to be influenced by other genetic and environmental factors. ${ }^{26}$

The CIAS1 mutation in CAPS is a gain-of-function mutation in cryopyrin, leading to activation of proinflammatory cytokines. IL-1, one of the proinflammatory cytokines activated in CAPS, is the primary target for drugs such as anakinra. It was originally thought that anakinra should be used in the treatment of NOMID patients with CIAS1 mutations only. However, a number of studies have demonstrated that anakinra is effective for treatment of the disease regardless of the gene defect present. For example, recent work by 
Lovell et al demonstrated improvement of NOMID symptoms in a patient with a normal CIAS1 gene sequence after treatment with anakinra. ${ }^{27}$ In another study by Hawkins et al, three patients were diagnosed with NOMID on clinical grounds and later revealed no CIAS1 mutations. After receiving anakinra, all three patients had resolution of fever, rash, joint inflammation, and headache, with normalization of C-reactive protein and erythrocyte sedimentation rate. ${ }^{28}$ These findings suggest that regardless of the upstream mechanism, such as CIAS1 defects, correction of the downstream effect including IL-1 $\beta$ overproduction ameliorates symptoms of the disease.

Another observation has been the presence of somatic mosaicism in patients with CIAS1-related disease. Tanaka et al were able to identify that NOMID/CINCA resulted from the mutant NLRP3 gene in these cases of somatic mosaicism, in which mutant cells accounted for $4.2 \%-35.8 \%$ of all blood cells. ${ }^{29}$

\section{Treatment}

\section{Traditional treatment}

Traditional treatment of CAPS has utilized anti-inflammatory agents such as glucocorticoids, nonsteroidal anti-inflammatory agents, antihistamines, and other immunosuppressive medications, all without significant success. While there have been modest improvements with high-dose oral corticosteroids and thalidomide, these agents have not been well tolerated due to their side effect profile. Because of the discovery that IL- $1 \beta$ is a potent proinflammatory cytokine which is upregulated by mutations in CIAS1, three new therapies have been identified which use different approaches to achieve the same goal.

\section{Anakinra: an IL-I receptor antagonist}

Exploration of IL-1 receptors started in 1993 with the introduction of anakinra, a recombinant, nonglycosylated version of IL-1 receptor antagonist (IL-1RA) prepared from cultures of genetically modified Escherichia coli using recombinant DNA technology. It consists of 153 amino acids and differs from native human IL-1RA in that it has the addition of a single methionine residue on its amino terminus. Since 1993, anakinra has been well studied and has been shown to control many diseases, including CAPS. Use of anakinra in different diseases is shown in a timeline in Figure 2. Anakinra currently dominates the field of IL-1 therapeutics, mostly because of its excellent safety profile and tolerability by pediatric and adult patients.

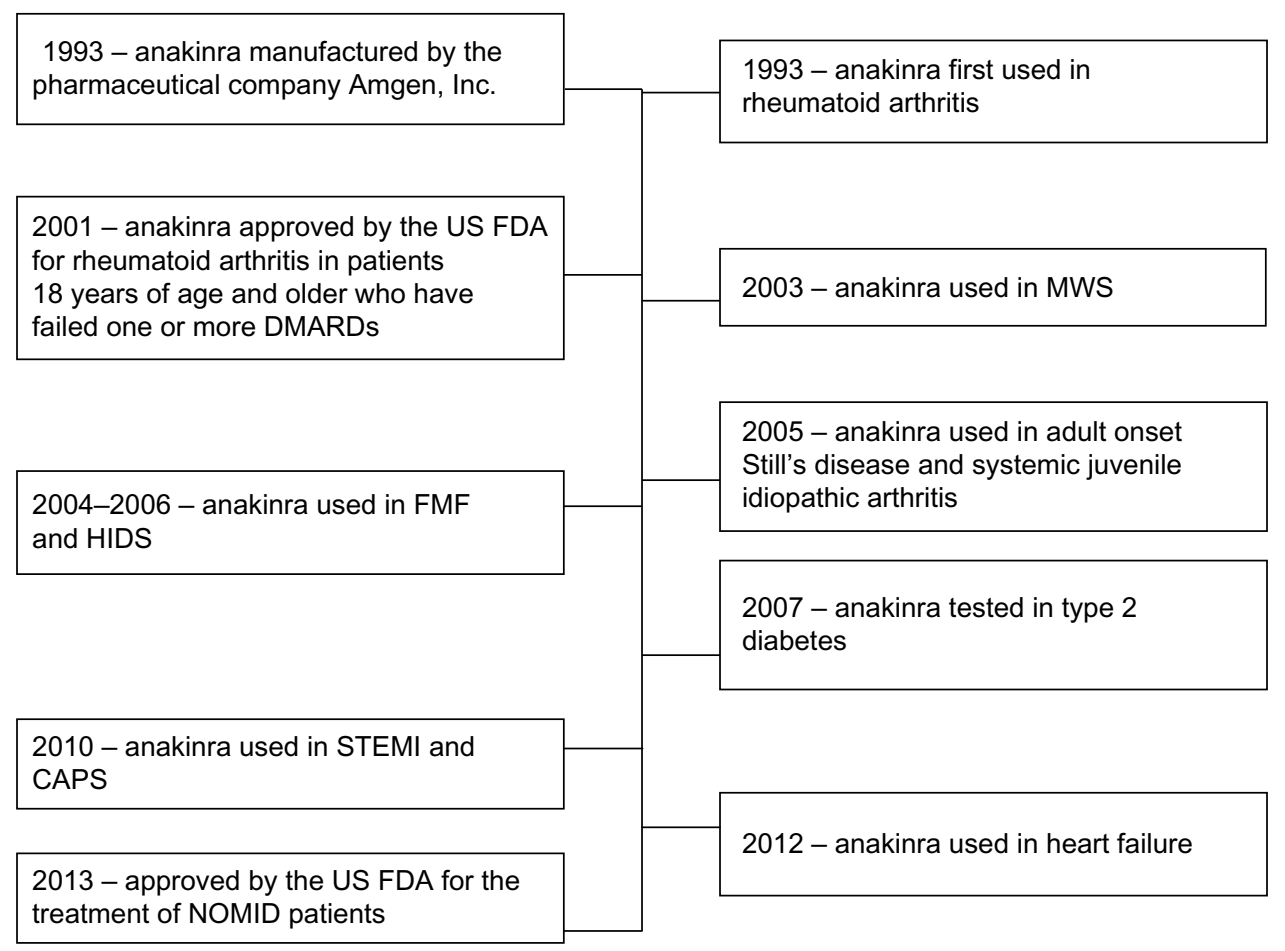

Figure 2 Timeline highlighting use of anakinra in various disease states.

Abbreviations: CAPS, cryopyrin-associated periodic syndromes; DMARDs, disease-modifying antirheumatic drugs; US FDA, US Food and Drug Administration; FMF, familial Mediterranean fever; HIDS, hyper-lgD syndrome; STEMI, ST elevation myocardial infarction; NOMID, neonatal onset multisystem inflammatory disease; MWS, Muckle-Wells syndrome. 
Anakinra was approved by the US Food and Drug Administration in January 2013 for the treatment of NOMID, and its use is expected to increase, given that it may have a beneficial effect in other disease states.

A role for anakinra in the treatment of CAPS has been evident in prevention of clinical symptoms and acute-phase elevations in patients with $\mathrm{FCAS},{ }^{30}$ an ability to induce complete remission in MWS, and resolution of symptoms including uveitis, rash, and fever in NOMID patients. ${ }^{31,32}$ The possibility of reducing the clinical symptoms of inflammation and improvement in quality of life makes this discovery extremely important. The safety and efficacy of anakinra have been tested in a number of studies, with promising results.

The short-term efficacy and rapid response to anakinra was first reported in a study by Hawkins et al, who followed three severely ill family members carrying NALP3/CIAS1 mutations. It was reported that clinical features such as rash, fever, conjunctivitis, and arthralgia improved within 4 hours after the first injection, and serologic remission was achieved within one week. The study also reported a rapid decrease in C-reactive protein levels and a resolution of hematologic abnormalities in all patients. ${ }^{33}$

Immediate resolution of clinical symptoms was also evident in a retrospective study by Leslie et al, who demonstrated complete remission of rash, fever, conjunctivitis, and rheumatic aches within 4-12 hours of starting anakinra in 15 patients treated for 39 months. These patients were treated starting with daily $100 \mathrm{mg}$ subcutaneous injections and then reduced to 20-50 $\mathrm{mg}$ to determine efficacy at lower doses. All patients improved on the lower doses, except for two children who continued to receive $100 \mathrm{mg}$ daily injections. Monthly measurements of C-reactive protein and serum amyloid A levels were reviewed, and demonstrated normalization within one week in all patients. In addition, nephrotic syndrome remitted within 8-33 months in three patients with AA amyloidosis. ${ }^{34}$

In a prospective study, Goldbach-Mansky et al demonstrated improvement in symptoms such as urticarial-like rash and acute-phase reactants, in addition to improvements in hearing and neurosensory deficits in 18 children with CINCA/ NOMID (12 with identifiable CIAS1 mutations) followed for 6 months. The treatment was able to effectively control most of the clinical manifestations, including chronic meningitis and papilledema. Abnormal cochlear enhancement was detected in 17 patients on magnetic resonance imaging, and although 13 of these patients had improvement of cochlear enhancement, one had a regression and three had no improvement after 3 months of therapy. Primary outcomes were decreases in C-reactive protein, erythrocyte sedimentation rate, and serum amyloid A levels, as well as daily improvement in ratings of symptoms at 3 months, all of which also remained low at 6 months. In total, eight of 18 patients showed remission of inflammatory symptoms after 3 months and the remaining ten patients after 6 months. Furthermore, treatment with anakinra reduced secretion of IL-1 $\beta$ from cultured peripheral blood mononuclear cells after 6 months of therapy. ${ }^{32}$

Not only has anakinra been useful in NOMID patients, but it has also shown improvement in patients with other CAPS disorders. In a study by Kuemmerle-Deschner et al, anakinra proved to be an effective and safe therapy in 12 children and adults with severe MWS over a median of 11 (5-14) months. There was rapid resolution of constitutional symptoms, severe disease-associated fatigue, and musculoskeletal, skin, and eye symptoms after 2 weeks of therapy. Classical markers of inflammation improved in the majority of these patients. The erythrocyte sedimentation rate normalized in all but one patient (92\%), and the C-reactive protein value normalized in $45 \%$ of those who had an elevation at baseline. While improvement in erythrocyte sedimentation rate was detectable as early as 2 weeks and then plateaued, C-reactive protein levels continued to improve further until the last follow-up visit. ${ }^{35}$

Similar observations were reported in a study by Hoffman et al, in which anakinra was able to prevent cold-associated acute inflammation in four patients with FCAS subjected to cold challenge. When subjected to cold challenge without anakinra treatment, the patients with FCAS developed low grade fever, rash, and arthralgia starting 1-2 hours after cold challenge. The patients with FCAS treated with anakinra before cold challenge did not develop any of the signs or symptoms that they had developed during the previous cold challenge. These patients did develop a mild rash after the initial dose, but had no symptoms after the second dose and remained symptom-free for 24-48 hours. ${ }^{30}$

Given that use of IL-1RA is relatively new, its efficacy and safety on a long-term basis is not well documented. Several studies have assessed the long-term effects of IL-1 antagonists in different phenotypes of CAPS patients, including NOMID/CINCA. Lepore et al demonstrated the longterm effects and sustainability of control of inflammatory manifestations in ten CINCA patients who were followed for 26-42 months. They demonstrated complete control of the inflammatory manifestations, such as skin rash, arthritis/ 
arthralgia, elevated acute-phase reactants, and inflammatory anemia for up to almost 5 years of follow-up. Continuous treatment with anakinra completely controlled some of the neurologic manifestations such as chronic meningitis and headache (although control of papilledema and amelioration of hearing loss was not achieved). ${ }^{36}$ In a study by Neven et al, the long-term effects of anakinra for up to 26-42 months have also been demonstrated in treating a broad range of systemic manifestations, neurologic involvement, and growth parameters. The magnitude of these responses was dosedependent, with improvement in constitutional symptoms such as fever, rash, and arthralgia at $1 \mathrm{mg} / \mathrm{kg}$ /day. Higher doses (2-3 mg/kg/day) were necessary in five patients with persistent headache and papilledema after one year of therapy in order to achieve ongoing normalization of biologic inflammation marker levels and decreases in central nervous system inflammation. ${ }^{10}$ However, central nervous system inflammation normalized in only one patient, with improvement and stabilization in a total of four patients. These data indicate that long-term remission is possible, but this is difficult to achieve in severe cases of NOMID.

Leslie et al also demonstrated a positive outcome in 15 MWS patients followed for a median of 17 (1-39) months. All patients showed a sustained response to drug therapy without dosage adjustments to prevent recurrence of symptoms. There was significant improvement in renal disease measured by scintigraphic scan for amyloidosis. $^{34}$

Based on the studies to date, it is clear that anakinra is a major advance in the management of NOMID and other CAPS phenotypes. To date, there have been very few side effects demonstrated with administration of anakinra. Local injection site reactions, including itching, swelling and redness, have been reported to occur in $42 \%$ of patients. ${ }^{35}$ Similar rates of local site reactions were reported in the other studies mentioned above. ${ }^{32-34}$ However, injection site reactions are uncommon after the first month of therapy. Despite the success of anakinra, the short half-life of 4-6 hours requires daily subcutaneous injections. Treatment becomes a lifelong process, because drug withdrawal leads to disease flares. As discussed below, other IL-1-blocking agents with longer half-lives are also being developed and tested with success.

\section{Rilonacept: IL-I trap}

Rilonacept, an IL-1 trap cytokine inhibitor, has been used effectively in the treatment of many diseases. Rilonacept utilizes cytokine trap technology, which allows for effective inhibition due to multicomponent "traps" containing binding sites for the cytokine as well as their accessory proteins. Cytokine traps have been very effective in autoinflammatory diseases because they bind to their respective cytokines with high affinity. ${ }^{37}$ Rilonacept was first approved by the US Food and Drug Administration in 2008 specifically for FCAS and MWS patients over the age of 11 years. The drug has a half-life of 8.6 days, which makes weekly injections an improvement over daily injections of anakinra. The safety and efficacy of weekly subcutaneous injections (160 mg/week) of rilonacept have recently been evaluated in patients with FCAS and MWS. The efficacy of treatment was confirmed in a one-year study, with five FCAS patients having confirmed mutations. ${ }^{38}$ All five patients showed improvement of symptoms, with the maximum improvement at days 6-10. All patients were given doses of $160 \mathrm{mg}$ weekly, although remission did not occur in four patients, who were then given doses of $320 \mathrm{mg}$. All five patients tolerated the drug very well with no injection site reactions, and showed long-term efficacy at the 2-year follow-up.

In two sequential, double-blind, placebo-controlled trials of patients with FCAS or MWS, all patients had positive CIAS1 gene mutations. In the first study, a randomized 6-week trial, 47 patients received either rilonacept at a dose of $160 \mathrm{mg} /$ week or placebo. The patients receiving the drug showed a decrease in disease activity score of $84 \%$ compared with $13 \%$ in patients receiving placebo. In the second study, patients were enrolled in a 9-week, single-blind treatment period followed by a 9-week, double-blind withdrawal period. Patients on rilonacept therapy during the "withdrawal" period demonstrated improved efficacy compared with the group on placebo. ${ }^{39}$ Patients treated with rilonacept had mild injection site reactions.

\section{Canakinumab: IL-I $\beta$ monoclonal antibody}

Canakinumab is a human IL- $1 \beta$ monoclonal antibody with very good efficacy in the treatment of CAPS patients. It has a long half-life of 26 days, which allows for a dosing schedule of subcutaneous injections every 8 weeks. This dosing schedule is favorable compared with that of anakinra (daily) and rilonacept (weekly). Canakinumab was approved by the US Food and Drug Administration in 2009 for use in CAPS patients over 4 years of age. The first open-label clinical trial for canakinumab was completed in 2008 in seven mutation-confirmed CAPS patients. In these patients, IL- $1 \beta$ production fell to levels similar to those seen in unaffected healthy controls after 8 weeks of therapy. ${ }^{40}$ 
In another double-blind, randomized trial in 2008, 35 CAPS patients (all with confirmed NLRP3 mutations), enrolled in a three-part, 48-week trial to receive $150 \mathrm{mg}$ of canakinumab every 8 weeks. In the first part, 34 of the 35 patients had a complete response in as little as 8 days, which was confirmed by physician assessment of disease activity and rash, as well as C-reactive protein and serum amyloid A levels. In the second part of the study, 15 patients received the drug and 16 patients received placebo. All 15 patients in the treatment group experienced remission of symptoms, while 13 of those in the placebo group experienced disease flares and increased C-reactive protein. In the third part, the 31 patients showed sustained clinical remission with minimal disease activity, disappearance of rash, and normalization of C-reactive protein and serum amyloid A levels. Complete remission was seen in $97 \%$ of the patients on treatment, and randomization to placebo led to disease flares in $81 \%$ of patients, whereas all patients who received the drug remained in remission. Injection site reactions occurred in up to $9 \%$ of patients, and up to $14 \%$ of the patients developed vertigo. $^{41}$

\section{Side effects of biological modulators of IL- I/IL-I R}

The primary side effects of anakinra in NOMID have been reported to be local injection site reactions, including redness, rash, itchiness, or pain. Other skin reactions such as bruising or bleeding can occur, but are very rare. Skin reactions usually resolve after $1-2$ weeks. Biological modulators carry a risk of infection, and this has been reported when anakinra is used to treat rheumatoid arthritis but not NOMID. Other reactions may include headaches, vomiting, arthralgias, pyrexia, and nasopharyngitis. The most common side effects of canakinumab in the treatment of CAPS include nasopharyngitis, diarrhea, nausea, influenza, and headache. A similar side effect profile was also described with rilonacept, with injection site reactions being the most common adverse effect.

\section{Discussion}

Remarkable advances have been made in our understanding of the molecular and genetic pathways responsible for the clinical phenotypes of NOMID. Studying patients with NOMID has allowed us to define the extent of disease damage caused by overproduction and secretion of IL- $1 \beta$ due to mutations in the CIAS1 gene. The discovery of these gene mutations has led to major advances in the treatment of this rare disease and led to rapid resolution of its inflammatory manifestations. IL-1 blocking agents have been found to be helpful not only in NOMID and other milder forms of CAPS, but also in other IL-1-mediated diseases, such as familial Mediterranean fever, gout/pseudogout, osteoarthritis, rheumatoid arthritis, type 2 diabetes mellitus, pyoderma gangrenosum, and other diseases as noted in Table 2. Numerous studies have shown positive effects of anakinra in patients with the above disorders. Significant studies are described in Table 3. Anakinra offers an ideal treatment option for many of these diseases due to its excellent safety profile and ease of administration subcutaneously or intravenously. The short half-life of 4-6 hours offers an additional safety benefit and allows for short-term use.

Studies of patients with NOMID have been imperative in characterizing the extent of organ-specific inflammatory manifestations. The disease severity in NOMID is extreme and requires close monitoring of the involved organs and aggressive treatment to control both systemic and organspecific manifestations. It is important for patients with NOMsID to receive an early diagnosis and prompt treatment, because cumulative inflammation can lead to progressive and permanent organ damage. Lepore et $\mathrm{al}^{36}$ reported on patients who received an early diagnosis and prompt treatment, and had not shown any new disease-related manifestations such

Table 2 Examples of conditions treated by anakinra

Inflammatory diseases
Gout and pseudogout
Type 2 diabetes
Pustular psoriasis
Sjögren's syndrome
Joint, bone, and muscle diseases
Rheumatoid arthritis
Ankylosing spondylitis
Erosive osteoarthritis of the hand
Osteomyelitis
Hereditary systemic autoinflammatory disease
Cryopyrin-associated periodic syndrome
Hyper-lgD syndrome
Familial Mediterranean fever
Periodic fever, aphthous stomatitis, pharyngitis, and adenitis
System inflammatory diseases
Systemic juvenile idiopathic arthritis
Adult onset Still's disease
Schnitzler syndrome
Behçet's disease
Synovitis, acne, pustulosis, hyperostosis, osteitis




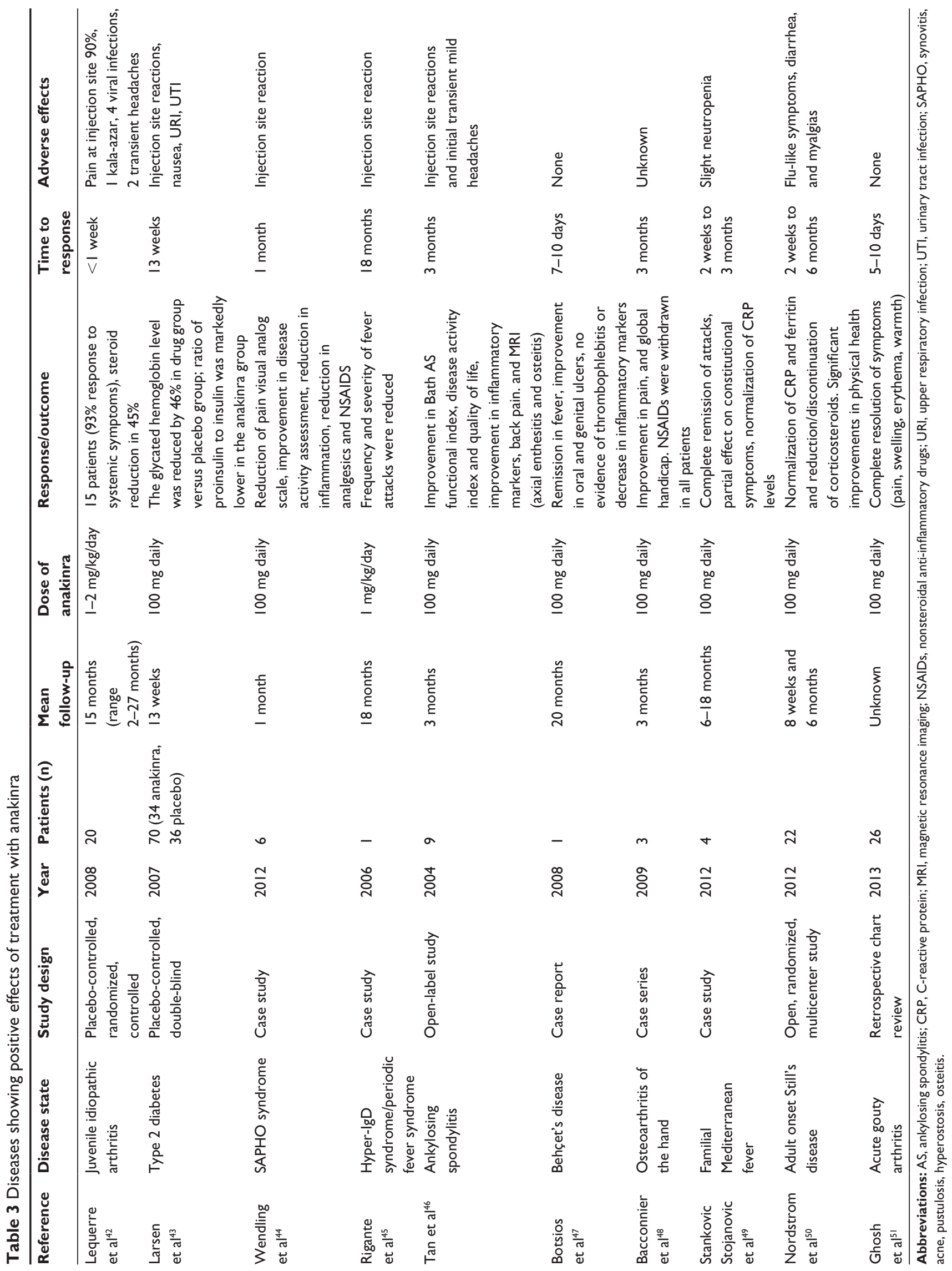


as hearing loss, papilledema, chronic meningitis, or mental retardation. These observations illustrate the need for prompt recognition and treatment of all CAPS phenotypes, including NOMID.

As demonstrated in the many studies of diseases treated by anakinra, the upstream mechanism or gene defect in a disease does not have as much influence as the downstream effects, including IL-1 $\beta$ overproduction. In NOMID syndrome, mutations in CIAS1 are found in about $50 \%$ of patients, and appear to be only one of the underlying genetic defects in this group of diseases. Recent studies suggest that treatment of patients with normal CIAS1 genetic sequencing may be successful by correction of the downstream effects (IL-1 $\beta$ overproduction) regardless of the upstream mechanism (CIAS1 defect). This imbalance of IL-1 $\beta$ production can be alleviated by administration of anakinra. Anakinra has been shown to achieve rapid and sustained clinical remission for the majority of CAPS patients, including NOMID syndrome, and many other disease states.

\section{Disclosure}

The authors report no conflicts of interest in this work.

\section{References}

1. Stojanov S, Kastner DL. Familial autoinflammatory diseases: genetics, pathogenesis and treatment. Curr Opin Rheumatol. 2005;17(5): 586-599.

2. Dhimolea E. Interleukin-1beta inhibitors for the treatment of cryopyrinassociated periodic syndrome. Appl Clin Genet. 2011;4:21-27.

3. Cuisset L, Jeru I, Dumont B, et al. Mutations in the autoinflammatory cryopyrin-associated periodic syndrome gene: epidemiological study and lessons from eight years of genetic analysis in France. Ann Rheum Dis. 2011;70(3):495-499.

4. Damiano JS, Oliveira V, Welsh K, Reed JC. Heterotypic interactions among NACHT domains: implications for regulation of innate immune responses. Biochem J. 2004;381 Pt 1:213-219.

5. Montealegre Sanchez GA, Hashkes PJ. Neurological manifestations of the Mendelian-inherited autoinflammatory syndromes. Dev Med Child Neurol. 2009;51(6):420-428.

6. Stych B, Dobrovolny D. Familial cold auto-inflammatory syndrome (FCAS): characterization of symptomatology and impact on patients' lives. Curr Med Res Opin. 2008;24(6):1577-1582.

7. Hoffman HM, Wanderer AA, Broide DH. Familial cold autoinflammatory syndrome: phenotype and genotype of an autosomal dominant periodic fever. J Allergy Clin Immunol. 2001;108(4):615-620.

8. Muckle TJ, Wellsm. Urticaria, deafness, and amyloidosis: a new heredo-familial syndrome. $Q J$ Med. 1962;31:235-248.

9. Prieur AM, Griscelli C. Arthropathy with rash, chronic meningitis, eye lesions, and mental retardation. J Pediatr. 1981;99(1):79-83.

10. Neven B, Marvillet I, Terrada C, et al. Long-term efficacy of the interleukin-1 receptor antagonist anakinra in ten patients with neonatalonset multisystem inflammatory disease/chronic infantile neurologic, cutaneous, articular syndrome. Arthritis Rheum. 2010;62(1):258-267.

11. Hoffman HM, Mueller JL, Broide DH, Wanderer AA, Kolodner RD. Mutation of a new gene encoding a putative pyrin-like protein causes familial cold autoinflammatory syndrome and Muckle-Wells syndrome. Nat Genet. 2001;29(3):301-305.
12. Sutterwala FS, Ogura Y, Szczepanik M, et al. Critical role for NALP3/ CIAS1/Cryopyrin in innate and adaptive immunity through its regulation of caspase-1. Immunity. 2006;24(3):317-327.

13. Agostini L, Martinon F, Burns K, McDermott MF, Hawkins PN, Tschopp J. NALP3 forms an IL-1beta-processing inflammasome with increased activity in Muckle-Wells autoinflammatory disorder. Immunity. 2004;20(3):319-325.

14. Bauernfeind F, Ablasser A, Bartok E, et al. Inflammasomes: current understanding and open questions. Cell Mol Life Sci. 2011;68(5): 765-783.

15. Martinon F, Petrilli V, Mayor A, Tardivel A, Tschopp J. Gout-associated uric acid crystals activate the NALP3 inflammasome. Nature. 2006;440(7081):237-241

16. Mariathasan S, Weiss DS, Newton K, et al. Cryopyrin activates the inflammasome in response to toxins and ATP. Nature. 2006;440(7081):228-232

17. Feldmeyer L, Keller M, Niklaus G, Hohl D, Werner S, Beer HD. The inflammasome mediates UVB-induced activation and secretion of interleukin-1beta by keratinocytes. Curr Biol. 2007;17(13):1140-1145.

18. Thomas PG, Dash P, Aldridge JR Jr, et al. The intracellular sensor NLRP3 mediates key innate and healing responses to influenza A virus via the regulation of caspase-1. Immunity. 2009;30(4):566-575.

19. Duncan JA, Gao X, Huang MT, et al. Neisseria gonorrhoeae activates the proteinase cathepsin $\mathrm{B}$ to mediate the signaling activities of the NLRP3 and ASC-containing inflammasome. J Immunol. 2009; 182(10): 6460-6469.

20. Manji GA, Wang L, Geddes BJ, et al. PYPAF1, a PYRIN-containing Apaf1-like protein that assembles with ASC and regulates activation of NF-kappa B. J Biol Chem. 2002;277(13):11570-11575.

21. Mariathasan S, Newton K, Monack DM, et al. Differential activation of the inflammasome by caspase-1 adaptors ASC and Ipaf. Nature. 2004;430(6996):213-218

22. Federici S, Caorsi R, Gattorno M. The autoinflammatory diseases. Swiss Med Wkly. 2012;142:w13602.

23. Dode C, Le Du N, Cuisset L, et al. New mutations of CIAS1 that are responsible for Muckle-Wells syndrome and familial cold urticaria: a novel mutation underlies both syndromes. Am J Hum Genet. 2002;70(6):1498-1506.

24. Janssen R, Verhard E, Lankester A, Ten Cate R, van Dissel JT. Enhanced interleukin-1beta and interleukin-18 release in a patient with chronic infantile neurologic, cutaneous, articular syndrome. Arthritis Rheum. 2004;50(10):3329-3333.

25. Aksentijevich I, Nowak M, Mallah M, et al. De novo CIAS1 mutations, cytokine activation, and evidence for genetic heterogeneity in patients with neonatal-onset multisystem inflammatory disease (NOMID): a new member of the expanding family of pyrin-associated autoinflammatory diseases. Arthritis Rheum. 2002;46(12):3340-3348.

26. Aksentijevich I, D Putnam C, Remmers EF, et al. The clinical continuum of cryopyrinopathies: novel CIAS1 mutations in North American patients and a new cryopyrin model. Arthritis Rheum. 2007;56(4):1273-1285.

27. Lovell DJ, Bowyer SL, Solinger AM. Interleukin-1 blockade by anakinra improves clinical symptoms in patients with neonatal-onset multisystem inflammatory disease. Arthritis Rheum. 2005;52(4):1283-1286.

28. Hawkins PN, Bybee A, Aganna E, McDermott MF. Response to anakinra in a de novo case of neonatal-onset multisystem inflammatory disease. Arthritis Rheum. 2004;50(8):2708-2709.

29. Tanaka T, Takahashi K, Yamane M, et al. Induced pluripotent stem cells from CINCA syndrome patients as a model for dissecting somatic mosaicism and drug discovery. Blood. 2012;120(6):1299-1308.

30. Hoffman HM, Rosengren S, Boyle DL, et al. Prevention of coldassociated acute inflammation in familial cold autoinflammatory syndrome by interleukin-1 receptor antagonist. Lancet. 2004;364(9447): 1779-1785.

31. Rynne M, Maclean C, Bybee A, McDermott MF, Emery P. Hearing improvement in a patient with variant Muckle-Wells syndrome in response to interleukin 1 receptor antagonism. Ann Rheum Dis. 2006;65(4):533-534. 
32. Goldbach-Mansky R, Dailey NJ, Canna SW, et al. Neonatal-onset multisystem inflammatory disease responsive to interleukin-1beta inhibition. N Engl J Med. 2006;355(6):581-592.

33. Hawkins PN, Lachmann HJ, Aganna E, McDermott MF. Spectrum of clinical features in Muckle-Wells syndrome and response to anakinra. Arthritis Rheum. 2004;50(2):607-612.

34. Leslie KS, Lachmann HJ, Bruning E, et al. Phenotype, genotype, and sustained response to anakinra in 22 patients with autoinflammatory disease associated with CIAS-1/NALP3 mutations. Arch Dermatol. 2006;142(12):1591-1597.

35. Kuemmerle-Deschner JB, Tyrrell PN, Koetter I, et al. Efficacy and safety of anakinra therapy in pediatric and adult patients with the autoinflammatory Muckle-Wells syndrome. Arthritis Rheum. 2011;63(3): 840-849.

36. Lepore L, Paloni G, Caorsi R, et al. Follow-up and quality of life of patients with cryopyrin-associated periodic syndromes treated with anakinra. J Pediatr. 2010;157(2):310-315. e311.

37. Economides AN, Carpenter LR, Rudge JS, et al. Cytokine traps: multi-component, high-affinity blockers of cytokine action. Nat Med. 2003;9(1):47-52.

38. Goldbach-Mansky R, Shroff SD, Wilson M, et al. A pilot study to evaluate the safety and efficacy of the long-acting interleukin-1 inhibitor rilonacept (interleukin-1 Trap) in patients with familial cold autoinflammatory syndrome. Arthritis Rheum. 2008;58(8):2432-2442.

39. Hoffman HM, Throne ML, Amar NJ, et al. Efficacy and safety of rilonacept (interleukin-1 Trap) in patients with cryopyrin-associated periodic syndromes: results from two sequential placebo-controlled studies. Arthritis Rheum. 2008;58(8):2443-2452.

40. Lachmann HJ, Lowe P, Felix SD, et al. In vivo regulation of interleukin 1 beta in patients with cryopyrin-associated periodic syndromes. $J$ Exp Med. 2009;206(5):1029-1036.

41. Lachmann HJ, Kone-Paut I, Kuemmerle-Deschner JB, et al. Use of canakinumab in the cryopyrin-associated periodic syndrome. $N$ Engl J Med. 2009;360(23):2416-2425.
42. Lequerre T, Quartier P, Rosellini D, et al. Interleukin-1 receptor antagonist (anakinra) treatment in patients with systemic-onset juvenile idiopathic arthritis or adult onset Still disease: preliminary experience in France. Ann Rheum Dis. 2008;67(3):302-308.

43. Larsen CM, Faulenbach M, Vaag A, et al. Interleukin-1-receptor antagonist in type 2 diabetes mellitus. $N$ Engl J Med. 2007;356(15) $1517-1526$.

44. Wendling D, Prati C, Aubin F. Anakinra treatment of SAPHO syndrome: short-term results of an open study. Ann Rheum Dis. 2012;71(6): $1098-1100$

45. Rigante D, Ansuini V, Bertoni B, et al. Treatment with anakinra in the hyperimmunoglobulinemia D/periodic fever syndrome. Rheumatol Int. 2006;27(1):97-100.

46. Tan AL, Marzo-Ortega H, O’Connor P, Fraser A, Emery P, McGonagle D. Efficacy of anakinra in active ankylosing spondylitis: a clinical and magnetic resonance imaging study. Ann Rheum Dis. 2004;63(9):1041-1045.

47. Botsios C, Sfriso P, Furlan A, Punzi L, Dinarello CA. Resistant Behcet disease responsive to anakinra. Ann Intern Med. 2008;149(4) 284-286.

48. Bacconnier L, Jorgensen C, Fabre S. Erosive osteoarthritis of the hand: clinical experience with anakinra. Ann Rheum Dis. 2009;68(6):1078-1079.

49. Stankovic Stojanovic K, Delmas Y, Torres PU, et al. Dramatic beneficial effect of interleukin-1 inhibitor treatment in patients with familial Mediterranean fever complicated with amyloidosis and renal failure. Nephrol Dial Transplant. 2012;27(5):1898-1901.

50. Nordstrom D, Knight A, Luukkainen R, et al. Beneficial effect of interleukin 1 inhibition with anakinra in adult-onset Still's disease. An open, randomized, multicenter study. J Rheumatol. 2012;39(10): 2008-2011.

51. Ghosh P, Cho M, Rawat G, Simkin PA, Gardner GC. Treatment of acute gouty arthritis in complex hospitalized patients with anakinra. Arthritis Care Res (Hoboken). 2013;65(8):1381-1384.
Open Access Rheumatology Research and Reviews

\section{Publish your work in this journal}

Open Access Rheumatology Research and Reviews is an international peer-reviewed, open access journal, publishing all aspects of clinical and experimental rheumatology in the clinic and laboratory including the following topics: Pathology, pathophysiology of rheumatological diseases; Investigation, treatment and management of rheumatological

\section{Dovepress}

diseases; Clinical trials and novel pharmacological approaches for the treatment of rheumatological disorders. The manuscript management system is completely online and includes a very quick and fair peerreview system, which is all easy to use. Visit http://www.dovepress.com/ testimonials.php to read real quotes from published authors. 